\title{
Thromboembolism during coiling of intracranial aneurysms: predictors and clinical outcome
}

\author{
Damian Kocur ${ }^{1}$, Piotr Paździora ${ }^{1}$, Nikodem Przybyłko' ${ }^{1}$, Wojciech Kukier ${ }^{1}$, Jan Baron ${ }^{2}$, Adam Rudnik ${ }^{1}$ \\ ${ }^{1}$ Department of Neurosurgery, School of Medicine in Katowice, Medical University of Silesia, Katowice, Poland \\ ${ }^{2}$ Department of Radiology and Nuclear Medicine, School of Medicine in Katowice, Medical University of Silesia, Katowice, Poland \\ Videosurgery Miniinv 2020; 15 (2): 319-328 \\ DOI: https://doi.org/10.5114/wiitm.2019.89118
}

\begin{abstract}
Introduction: Thromboembolism is one of the most serious complications associated with coil embolization therapy. Aim: To identify predisposing factors for thromboembolic complications, as well as to determine whether thromboembolism has an impact on clinical outcome.

Material and methods: From February 2008 to March 2015, 273 consecutive patients were treated at our institution via endovascular coil embolization. Patient medical records were reviewed with an emphasis on procedure description, potential risk factors and clinical outcomes related to thromboembolism. Thromboembolic incidents occurred in 19 (6.9\%) cases. Multivariate logistic regression models were used to determine independent predictors of thromboembolism. Clinical outcome was analyzed using the Glasgow Outcome Scale (GOS).

Results: Multivariate analysis showed that subarachnoid hemorrhage was an independent risk factor for thromboembolic complications ( $p=0.003 ; O R=4.4 ; 95 \% \mathrm{Cl}: 1.67-12.02$ ). The difference in frequency of perioperative mortality (GOS 1) in patients with thromboembolism and without thromboembolism was not statistically significant ( $p=0.22$ ). The differences in frequencies of severe disability (GOS 2-3) and moderate to low disability (GOS 4-5) between patients with thromboembolism and without thromboembolism were statistically significant in the general study population $(p<0.05)$.

Conclusions: Subarachnoid hemorrhage is an independent predictor of thromboembolic complications associated with endovascular coiling of cerebral aneurysms. Thromboembolism is associated with significantly increased risk of morbidity, and it affects to a certain extent the periprocedural mortality.
\end{abstract}

Key words: complications, embolization, endovascular aneurysm repair.

\section{Introduction}

Thromboembolic events (TEES) represent one of the most serious complications of embolization therapy of cerebral aneurysms [1-3]. The incidence of TEEs reported by previous studies ranges from $2 \%$ to $77 \%$, and the possibility of permanent neurologic disability and death associated with TEEs is up to $8.4 \%$ [3-14]. To decrease the overall rate of coiling-related TEEs and reduce their unfavorable clini- cal consequences, it is desirable to identify the risk factors that predispose to TEEs and that should be considered when choosing embolization as a form of treatment of intracranial aneurysms [2]. So far, a number of risk factors for TEEs have been described in the literature. However, a large proportion of the studies are limited to a selected patient population and the results are frequently contradictory.

Among risk factors for TEEs one may distinguish: procedure-related factors (procedure time, proce-

\section{Address for correspondence}

Dr. Damian Kocur, Department of Neurosurgery, School of Medicine in Katowice, Medical University of Silesia, 14 Medyków St,

40-752 Katowice, Poland, phone: +48 507230 509, e-mail: damkocur@gmail.com 
dure methods, number of coils inserted, extent of procedure manipulations, operator experience, etc.) and non-modifiable factors (patient age, aneurysm size and location, presence of subarachnoid hemorrhage (SAH), smoking, etc.) [15]. Identification of the former factors may be of importance for improving the operation technique, which is considered to have a high possibility of affecting the occurrence of TEEs [15]. By determining the latter factors one may recognize a group of high-risk patients and apply an appropriate preventive therapy.

It should also be noted that in the literature there are no existing unequivocally established methods for prevention of TEEs. At present, periprocedural antiplatelet agents are commonly administered; however, the optimal patient selection, preferred antiplatelet agent, and dosing protocol, including the dose, timing, and duration of antiplatelet application, are still under debate [12]. Moreover, variation in individual response to antiplatelet agents has sparked a great deal of research and controversy in the neuroendovascular area [16].

In view of this, TEEs seems to be a complex process with multifactorial etiology. Despite this, it may be hypothesized that the identification of independent and unquestionable risk factors for TEEs in combination with appropriate prophylaxis will allow the incidence of TEEs to be significantly decreased in the future.

\section{Aim}

The aim of the study was to report the characteristics of patients experiencing TEEs during attempted coil embolization of cerebral aneurysms as well as to identify independent risk factors predisposing to TEEs and to determine whether TEEs may affect the clinical outcome in patients undergoing coil embolization of intracranial aneurysms.

\section{Material and methods}

\section{Database and risk factors}

From February 2008 to March 2015, 331 endovascular coiling procedures were performed on 273 consecutive patients at our institution. Patient medical records were reviewed and patients with TEEs were identified. Repeated endovascular procedures in recurrent aneurysms were excluded from the study.
The analyzed risk factors for TEEs included sex, age, presence of hypertension, atherosclerosis, smoking, subarachnoid hemorrhage, vasospasm, clinical status on admission, stent-assisted coiling, aneurysm size, neck size, aspect ratio, dome-to-neck ratio, aneurysm location, duration of embolization procedure, and experience of operator.

\section{Endovascular technique}

The technique of embolization procedures was described in our previous report [17]. Anti-platelet premedication consisted of a $75 \mathrm{mg}$ loading dose of acetylsalicylic acid and an additional $75 \mathrm{mg}$ of clopidogrel whenever stenting (Neuroform, Boston Scientific) was anticipated. This therapy was administered 2-4 $\mathrm{h}$ before the endovascular procedure on patients after SAH and every single day for 5 days before the treatment in the case of patients with unruptured aneurysms. During the procedures, heparinized saline was continuously infused into the arterial line. Heparin was usually administered as a 3000-4000 IU bolus intravenously followed by $1000 \mathrm{lU} / \mathrm{h}$. If TEEs occurred, a $20 \mathrm{mg}$ dose of abciximab was administered intra-arterially in all but one case of the accompanying intraprocedural aneurysm rupture. In that case, a Penumbra revascularization device was used. In patients with TEEs control angiograms were carried out to check the patency of the occluded vessel and to demonstrate the grade of aneurysm occlusion. Brain computed tomography (CT) scans were always analyzed at the end of the procedures.

\section{Thromboembolism definition and classification}

Thromboembolism was defined as a luminal filling defect or no visualization of a distal artery on digital subtraction angiography [8, 11, 18, 19]. Thromboembolic complications were classified into two groups: occlusion of the proximal arterial trunk (i.e. internal carotid artery, anterior communicating artery, middle cerebral artery, or basilar artery); and occlusion of the distal branches.

\section{Recanalization classification}

The response to thrombolytic therapy was determined using the Thrombolysis In Cerebral Infarction ( $\mathrm{TICl}$ grading system that is based on angiographic features of the treated occluded vessels as follows: 
no changes in the distal flow $(\mathrm{TICl} 0)$, partial recanalization ( $\mathrm{TICl} 1$ and 2), or complete recanalization (TICI 3) [20].

\section{Aneurysm morphology}

The aneurysm morphology was assessed at a Philips Integris 3D-RA Release 4.2 workstation using three-dimensional digital subtraction angiography images that were retrieved from the archives. Aneurysm size was defined as the maximum distance between the neck and the dome of an aneurysm. Neck size was defined as the maximal longitudinal neck width. Aspect ratio was defined as the maximal distance from the neck to the aneurysm dome to the maximal longitudinal neck width. Dome-to-neck ratio was defined as the aneurysm dome's maximal longitudinal diameter parallel with the neck plane to the maximal longitudinal neck width.

\section{Clinical results}

Clinical outcome was analyzed using the Glasgow Outcome Scale at discharge from hospital and during the last angiographic follow-up period. Patients without angiographic follow-up were interviewed by telephone if possible.

\section{Statistical analysis}

The statistical analysis was performed using SAS 9.4 software. The continuous data were presented as means \pm SDs, and categorical variables were expressed as frequency (percentage). To identify potential variables associated with TEEs, univariate regression analyses were performed. TEEs were used as the dependent variable in this model. The multivariate logistic regression model included covariates found to have a marginal association with TEEs in the univariate analysis (defined as $p<0.1$ ) and was performed using the backward regression method. ORs and $95 \% \mathrm{Cl}$ s were calculated. A $p$-value $<0.05$ was considered statistically significant.

For the purpose of the statistical analysis, the patients' clinical outcome was classified into the subgroups of low to moderate disability (patients with GOS 4 and 5), severe disability (patients with GOS 2 and 3) and death (patients with GOS 1). The cumulative frequency for TEEs was calculated for all aneurysms of these subgroups. Fisher's exact test was performed to test for an independence between the above-mentioned variables.

\section{Results}

\section{Patients and aneurysms}

A total of 273 patients (208 women) with a mean age of 54.01 years (range: $21-81$ years) were analyzed. There were 77 (28.2\%) patients after SAH and 196 (71.8\%) patients with unruptured aneurysms. The aneurysms were located in: the anterior communicating artery $(n=71)$, middle cerebral artery (MCA) $(n=52)$, the ophthalmic artery $(n=51)$, the posterior communicating artery $(n=38)$, the basilar artery $(n=28)$, the internal carotid artery $(n=20)$, the carotid terminus $(n=10)$, and the vertebral artery $(n=3)$. One hundred thirty-six patients were treated with standalone coiling and 137 patients with stent-assisted coiling. Mean aneurysm size was $6.7 \pm 3.8 \mathrm{~mm}$ (range: 1.6-29.4 mm), mean neck size was $4.8 \pm 2.2 \mathrm{~mm}$ (range: $1.4-15.9 \mathrm{~mm}$ ), mean aspect ratio was 1.4 \pm 0.7 (range: $0.3-5.5 \mathrm{~mm}$ ), and mean dome-to-neck ratio was $1.4 \pm 0.5$ (range: $0.6-3.5$ ). Detailed patient characteristics and aneurysm morphologies in TEE and non-TEE groups are presented in Table I.

\section{Incidence of TEEs}

The cumulative frequency of TEEs was 19 out of 273 endovascular procedures, which represented the TEE rate of $6.9 \%$. A summary of all TEE cases is depicted in Table II.

\section{Risk factors of TEEs}

Univariate analysis showed that patients after SAH were associated with TEE occurrence $(p=0.005)$. The cumulative frequency of TEEs was 12 out of $78(15.3 \%)$ in patients who previously experienced SAH. TEEs tended to occur more frequently in patients with a larger dome-to-neck ratio $(p=0.067)$. Multivariate analysis showed that SAH was an independent risk factor for TEEs ( $p=0.003$; $\mathrm{OR}=4.4 ; 95 \% \mathrm{Cl}: 1.67-12.02)$.

\section{Clinical outcome}

The cumulative frequency of perioperative mortality (GOS 1) was 2 out of $19(10.5 \%)$ in patients with TEEs and 11 out of $254(4.3 \%)$ in patients without TEEs. The difference was not statistically significant $(p=0.22)$.

The cumulative frequency of severe disability (GOS 2-3) was 5 out of 19 (26.3\%) in patients with TEEs and 12 out of $254(4.7 \%)$ in patients without 
Table I. Univariate analysis of factors affecting thromboembolism during endovascular coiling

\begin{tabular}{|c|c|c|c|c|}
\hline Variables & $\begin{array}{l}\text { Thromboembolism } \\
\qquad(n=19)\end{array}$ & $\begin{array}{l}\text { No thromboembolism } \\
\qquad(n=254)\end{array}$ & $P$-value & OR $(95 \% \mathrm{Cl})$ \\
\hline \multicolumn{5}{|l|}{ Clinical data: } \\
\hline Female & $14(73.7)$ & $194(76.4)$ & 0.790 & $0.86(0.29-2.50)$ \\
\hline Age [years] & $53.1 \pm 11.9$ & $54.1 \pm 11.0$ & 0.726 & $0.99(0.95-1.03)$ \\
\hline Hypertension & $7(36.8)$ & $123(48.4)$ & 0.445 & $0.68(0.25-1.81)$ \\
\hline Subarachnoid hemorrhage & $12(63.1)$ & $66(25.9)$ & 0.005 & $3.91(1.51-10.15)$ \\
\hline WFNS grade IV-V & $3(15.8)$ & $10(3.9)$ & 0.316 & $2.13(0.48-9.45)$ \\
\hline Fisher grade III-IV & $5(26.3)$ & $37(14.6)$ & 0.611 & $0.71(0.19-2.58)$ \\
\hline Smoking & $3(15.8)$ & $70(27.6)$ & 0.23 & $0.49(0.13-1.74)$ \\
\hline Atherosclerosis & $5(26.31)$ & $72(28.34)$ & 0.84 & $0.90(0.31-2.59)$ \\
\hline Vasospasm & $1(5.26)$ & $22(8.66)$ & 0.58 & $0.58(0.07-4.59)$ \\
\hline \multicolumn{5}{|l|}{ Aneurysm morphology: } \\
\hline Aneurysm size [mm] & $7.6 \pm 4.3$ & $6.7 \pm 3.8$ & 0.346 & $1.05(0.94-1.16)$ \\
\hline Neck size $[\mathrm{mm}]$ & $5.0 \pm 2.7$ & $4.9 \pm 2.3$ & 0.811 & $1.02(0.83-1.26)$ \\
\hline Aspect ratio & $1.6 \pm 0.6$ & $1.4 \pm 0.7$ & 0.284 & $1.37(0.76-2.45)$ \\
\hline Dome-to-neck ratio & $1.9 \pm 0.5$ & $1.6 \pm 0.7$ & 0.067 & $1.51(0.97-2.35)$ \\
\hline \multicolumn{5}{|l|}{ Aneurysm location: } \\
\hline Ophthalmic artery & $3(15.8)$ & $51(20.1)$ & 0.737 & $0.80(0.22-2.87)$ \\
\hline PComA & $2(10.5)$ & $36(14.1)$ & 0.659 & $0.71(0.15-3.21)$ \\
\hline Carotid terminus & 0 & $10(3.9)$ & 0.995 & 0 \\
\hline AComA & $6(31.6)$ & $65(25.6)$ & 0.567 & $1.34(0.49-3.67)$ \\
\hline MCA & $3(15.8)$ & 49 (19.3) & 0.708 & $0.78(0.21-2.79)$ \\
\hline Other & $5(26.3)$ & $46(18.1)$ & 0.395 & $1.61(0.55-4.70)$ \\
\hline \multicolumn{5}{|l|}{ Embolization aspects: } \\
\hline Stent-assisted coiling & $7(36.8)$ & $130(51.2)$ & 0.227 & $0.55(0.21-1.44)$ \\
\hline Operator experience [months] & $57.3 \pm 21.3$ & $55.07 \pm 24.5$ & 0.66 & $0.99(0.97-1.01)$ \\
\hline Embolization time [min] & $168.42 \pm 65.9$ & $22(8.66)$ & 0.58 & $0.58(0.07-4.59)$ \\
\hline
\end{tabular}

Continuous variables are depicted as mean \pm SD and categorical variables as number (\%). PComA - posterior communicating artery, AComA - anterior communicating artery, MCA - middle cerebral artery, GOS - Glasgow Outcome Score, WFNS - World Federation of Neurosurgical Societies.

TEEs. The difference was statistically significant $(p=0.0034)$.

The cumulative frequency of moderate to low disability (GOS 4-5) was 12 out of 19 (63.2\%) in patients with TEEs and 231 out of 254 (91\%) in patients without TEEs. The difference was statistically significant ( $p=0.0019)$.

\section{Discussion}

\section{Risk factors}

In the current study the multivariate logistic analysis revealed the presence of $\mathrm{SAH}$ as an independent predictor for TEEs during endovascular coiling of intracranial aneurysms. Previous obser- 


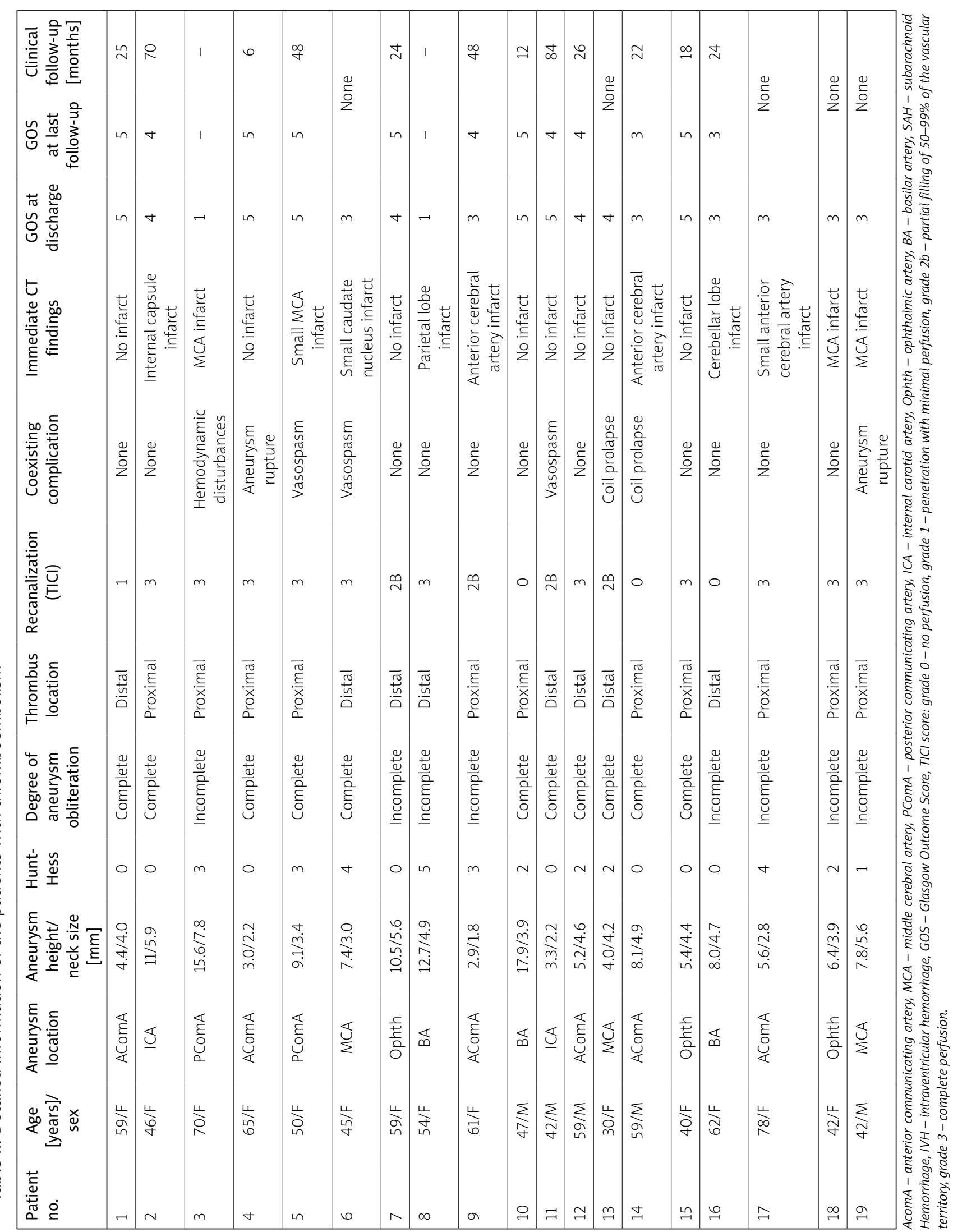


vational studies have suggested that SAH patients are at a higher risk of TEEs than patients with unruptured aneurysms $[12,21]$. There are also reports where the difference between incidences of patients with ruptured and unruptured aneurysms was not statistically significant in terms of TEEs $[15,19]$. The outcome in logistic regression analysis performed by Choi et al. demonstrated SAH at presentation as the sole independent risk factor for TEEs [22]. The authors analyzed 9 cases of procedure-related TEEs in a group of 285 anterior communicating artery aneurysms [22]. Our results are in line with the latter study; however, our study included all locations of cerebral aneurysms.

The higher incidence of TEEs in ruptured aneurysms in comparison to unruptured ones may be related to a limited use of preoperative antiplatelet therapy as well as vasospasm and hypercoagulability in the former group of patients $[4,6]$. The blood in the subarachnoid space catalyzes the release of the tissue factor into the systemic blood circulation, leading to thrombin activation and subsequently fibrin and platelet aggregation [4, 23]. The thromboembolism during the endovascular coiling may result from spontaneous activation of the coagulation system in SAH patients, as well as from endovascular material (e.g. catheters, stents) [4]. The presence of foreign materials placed during embolization, i.e. catheters, stents, and coils, in the aneurysm lumen and in the parent artery may be sufficient to induce a thromboembolism [4, 9, 24-26]. Additionally, during the procedure, an intimal injury of the arterial vessel from microcatheter displacement or stent insertion may lead to the release of procoagulant tissue factors, resulting in the formation of a platelet-rich thrombus [1, 6, 9, 25-28]. Apart from that, the irregular coil surface or coil protrusion into the vessel may result in vessel narrowing, leading to changes in blood flow. Then, thromboembolism is likely to happen at the interface of the coil and luminal blood [1, 3, 4, 8, 12, 28, 29].

As far as stents are concerned, in the literature, there is a disagreement whether the stent-assisted coiling is associated with a higher rate of TEEs $[10,30,31]$. Similarly, a controversy surrounds the history of hypertension, vasospasm, smoking, large aneurysm size, wide aneurysm neck, location of aneurysm on anterior communicating artery, middle cerebral artery, or basilar artery $[1,2,8,12,14-16$, $32,33]$. These risk factors for TEEs act through var- ious mechanisms. Hypertension and smoking promote cerebrovascular dysfunction [14]. In large aneurysms an increased volume of clot is induced by coiling, which may predispose to TEEs. In the case of wide-neck aneurysms the surface of coils at the level of the neck is greater than in small-neck aneurysms, which may lead to higher risk of TEEs [14]. Despite these above-mentioned potential causes of TEEs, our study demonstrated no relationship between these factors and the risk of TEEs.

According to Lee et al. the longer operation time and associated multiple procedural manipulations is the most effective factor an interventionist can modify to decrease the TEE rate during coil embolization of intracranial aneurysms [15]. Although these authors together with some previous researchers showed that longer interventional duration was significantly associated with TEEs, the outcomes of the present study have not confirmed it $[8,15,16]$. In addition to procedure time, we also demonstrated that operator experience is not a predictor for TEEs.

In the case of risk factors for TEEs one reason for the discrepancy between studies might be various perioperative antiplatelet protocols [34, 35]. In the literature, there is no consensus on the optimal protocol to prevent thromboembolism during endovascular embolization of cerebral aneurysms [4, 32]. There is currently no general agreement about optimal patient selection, preferred antiplatelet agent, and dosing protocol [12]. The most commonly applied means are systemic heparinization, heparinized saline, and antiplatelet therapy, but in the case of ruptured aneurysms, the use of a preventive protocol is limited in view of the potential increased risk of hemorrhagic complications [4, 23, 24, 36, 37]. Similar to many but not all practitioners, we applied in the present study dual antiplatelet therapy consisting of acetylsalicylic acid and a P2Y12 inhibitor (clopidogrel); however, the anticoagulation protocol differed in unruptured and ruptured aneurysms. In the case of patients with unruptured aneurysms we initiated the antiplatelets 5 days prior to the procedures. Patients following SAH could not be appropriately prepared and they received a single dose of antiplatelets directly before the operation. Due to the higher rate of TEEs in ruptured aneurysms, we suggest that our protocol in this group was inadequate, which is in line with previous studies implying dubious benefits of periprocedural antiplatelet therapy in SAH patients [12]. Due to the inadequate 
response to clopidogrel in a relatively high proportion of the population, two additional thienopyridine derivatives (prasugrel and ticagrelor) may be an option [26]. These agents, unlike clopidogrel, do not require activation via the cytochrome $\mathrm{P} 450$ system and, therefore, have a decreased risk of suboptimal platelet inhibition [26].

\section{Frequency and definitions}

The reported incidence of TEEs ranges very widely between $2 \%$ and $77 \%$ [4-12]. The $6.9 \%$ frequency of TEEs in the current study is at the lower end of this range. This may be due to the fact that the incidence of TEEs may be underestimated, as it is not always recognized, especially in the case of silent infarcts that are often detected on post-procedure diffusion-weighted magnetic resonance imaging (DWI) $[9,12,31,32]$. In view of incoherent definitions of TEEs in the literature, the incidence of TEEs may not always be comparable between studies [32].

The common definition of thromboembolism is "angiographic slow filling of a previously normally filling vessel or vessel dropout" [2, 3, 8, 11, 18, 19]. This may or may not be accompanied by deterioration of patients' condition [12, 23, 30]. According to some authors, TEEs without clinical evidence of cerebral ischemia are not recorded as complications and clinically "silent" infarcts are often identified on DWI [38]. These small high-intensity signals are found in more than $60 \%$ of patients undergoing embolization therapy and their presence indicates that microemboli may occur during endovascular embolization $[9,10$, $32,36]$. Thrombotic microemboli can originate from preexisting thrombus or intravascular thrombotic plaques that are displaced during the procedure; and gaseous microemboli can arise during catheter flushing guidewire exchange or contrast medium injection [9]. The clinical significance of microemboli detected as small DWI-positive lesions is yet to be determined, especially in long-term follow-up [9].

\section{Clinical outcome}

In the literature, once a thromboembolic event occurred, morbidity was up to $45 \%$ and mortality was up to $23 \%[3,13,14]$. In our study severe neurologic deficit was encountered in 5 of 19 (26.3\%) patients following TEEs and 2 of 19 (10.5\%) patients who died as a consequence of TEEs. According to previous observational reports, TEEs is the major cause of morbidity and mortality in patients undergoing endovascular embolization of intracranial aneurysms $[13,19]$. Fan et al. found in univariate analysis that patients with anterior communicating aneurysms who experienced TEEs during coiling have a significantly worse clinical course and an increased risk of mortality [2]. In our study, we observed that in the general population, TEEs have an influence on postoperative morbidity (Photo 1). There was a tendency for TEEs to affect the periprocedural mortality, but it did not achieve statistical significance. Different methodology and patient selection may be responsible for these inconsiderably heterogeneous results [2]. Moreover, disparate time of TEE recognition and effectiveness of TEE management may be factors influencing the various reduction of the morbidity and mortality rate [39]. Koebbe et al. stated that good condition at presentation and successful recanalization may be factors for increased survival following TEEs [40]. In the case of acute ischemic stroke, recanalization has been proven to be strongly associated with improved functional outcomes and reduced mortality [41]. It is also suggested that not only treatment effectiveness, but also time of TEE recognition is crucial in reducing the negative clinical sequelae of TEEs [39]. Based on our research, we may suggest that the aggressive treatment of TEEs is likely to decrease the rate of mortality. However, some individual cases of complete recanalization with subsequent inauspicious clinical results presented in the current study are supposed to be associated with delayed institution of appropriate rescue therapy. This indicates the important role of attention that should be directed to early detection of TEEs during coiling.

\section{Limitations}

One of the major limitations of the study is a different antiplatelet protocol in patients with unruptured and ruptured aneurysms. The probable inadequate application of antiplatelets in the latter subgroup of patients may have a substantial impact on the results.

In addition, the interindividual variability in the response to aspirin and clopidogrel was not included in the study. The presence of antiplatelet resistance may be associated with the higher risk of TEEs.

The incidence of TEEs was established based on digital subtraction angiography (DSA) instead of 
A

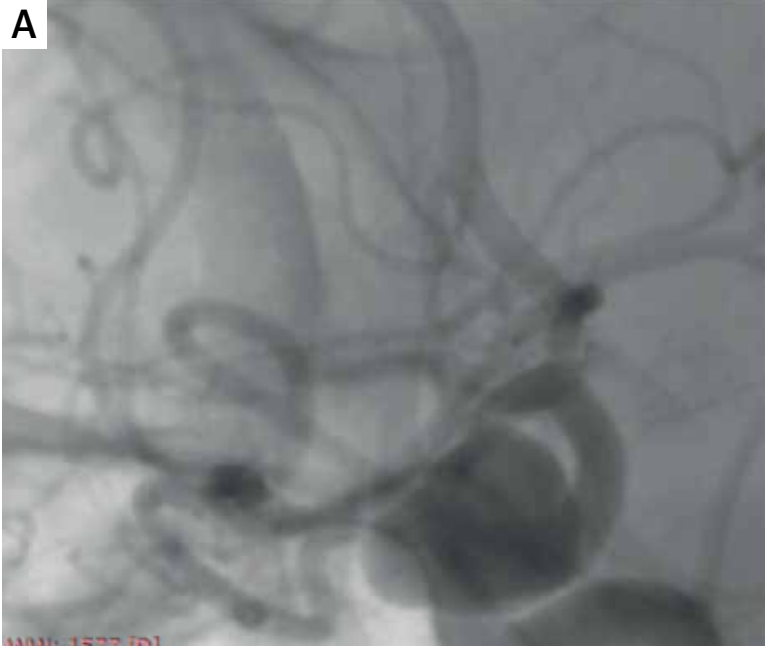

C

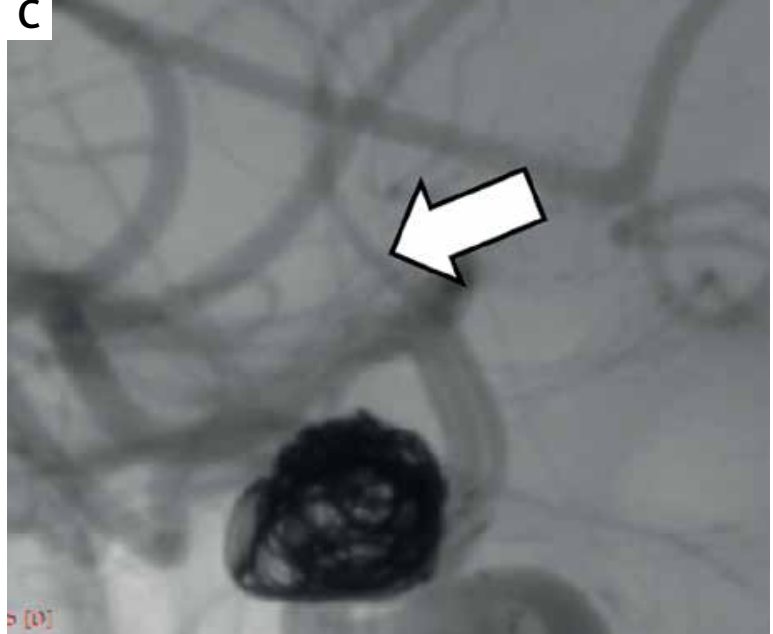

B

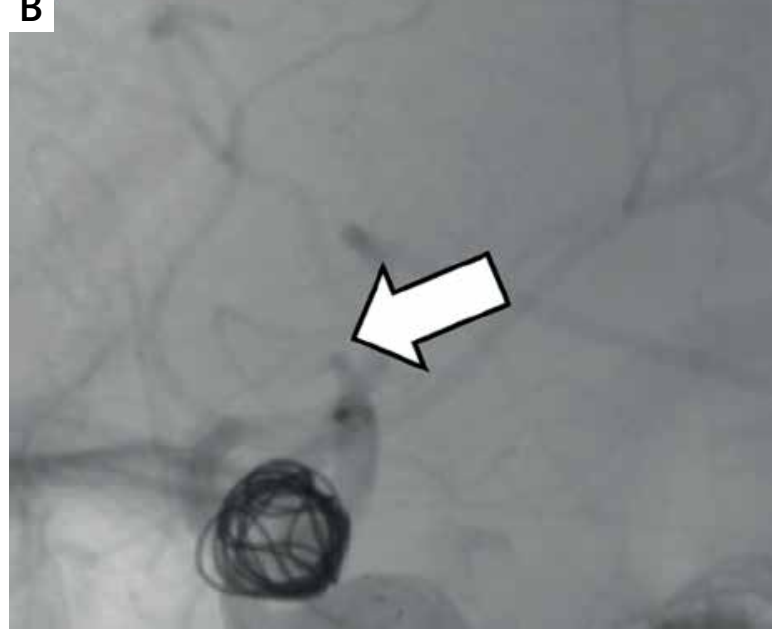

D

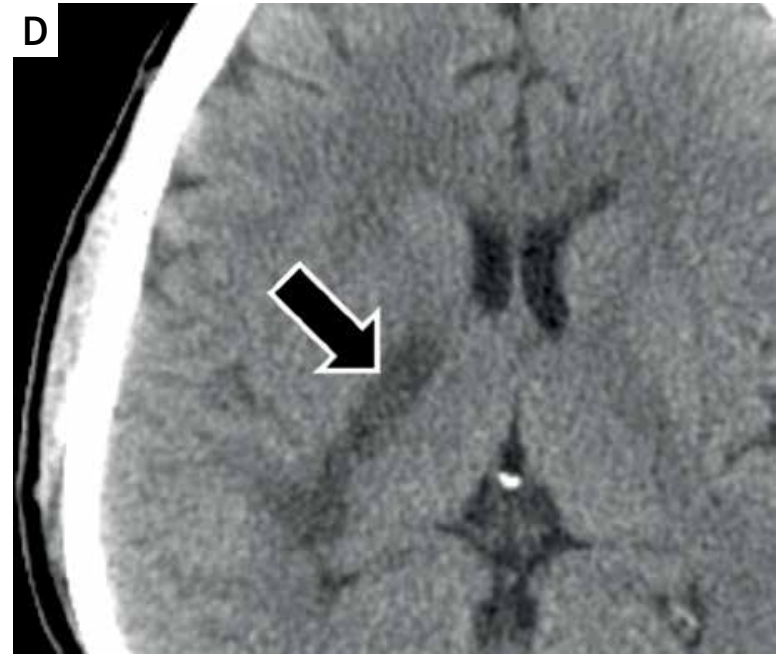

Photo 1. A 46-year-old woman was admitted to the hospital in good clinical condition. A - Lateral carotid angiogram showed the unruptured internal carotid artery aneurysm. B - A stent was placed in the internal carotid artery and after coiling, proximal occlusion of the ipsilateral middle cerebral artery occurred (white arrow). C - After administration of abciximab the vessel reopened (white arrow). Grade 3 in the Thrombolysis in Cerebral Infarction (TICI) scale was determined. D - A postoperative CT scan demonstrated internal capsule infarction (black arrow). The patient remained moderately disabled (GOS 4) within 70 months of clinical follow-up. According to the results of our study a thromboembolic incident during embolization is significantly associated with periprocedural morbidity $(p<0.005)$

DWI, which is more sensitive. Therefore, the rate of TEEs presented in the study may be undervalued.

The time of TEE recognition and the time of recanalization that may be associated with neurological recovery was not considered in the current study and these factors may have an impact on the different clinical outcomes.

Additionally, the study had a retrospective design and all the data were from a single neurovascular institution. There could be a patient selection bias, as factors that affect decision making for endovascular therapy were based on the center's experience and at times on patients' preferences.

\section{Conclusions}

The risk of TEEs detected by angiography during endovascular coil embolization is $6.9 \%$. Subarachnoid hemorrhage is an independent predictor for TEEs. The occurrence of this complication is associ- 
ated with significantly increased risk of morbidity, and it affects to a certain extent the periprocedural mortality.

\section{Conflict of interest}

\section{The authors declare no conflict of interest.}

\section{References}

1. Kim SH, Kim TG, Kong MH. Intra-arterial and intravenous tirofiban infusion for thromboembolism during endovascular coi embolization of cerebral aneurysm. J Korean Neurosurg Soc 2017; 60: 518-26.

2. Fan L, Lin B, Xu T, et al. Predicting intraprocedural rupture and thrombus formation during coiling of ruptured anterior communicating artery aneurysms. J Neurointerv Surg 2017; 9: 370-5.

3. Chen M. A checklist for cerebral aneurysm embolization complications. J Neurointerv Surg 2013; 5: 20-7.

4. Jun HS, Ahn JH, Kim JH, et al. Thrombus remnant despite intra-arterial thrombolysis for thrombus formation during endovascular treatment of ruptured cerebral aneurysms: does it harm? Interv Neuroradiol 2016; 22: 407-12.

5. Brinjikji W, McDonald JS, Kallmes DF, Cloft HJ. Rescue treatment of thromboembolic complications during endovascular treat ment of cerebral aneurysms. Stroke 2013; 44: 1343-7.

6. Ahn JH, Jun HS, Song JH, et al. Rescue mechanical thrombectomy using a retrievable stent for thromboembolic occlusion occurring during coil embolization of ruptured intracranial aneurysms. J Neurointerv Surg 2017; 9: 244-9.

7. Song JK, Niimi Y, Fernandez PM, et al. Thrombus formation during intracranial aneurysm coil placement: treatment with intra-arterial abciximab. AJNR Am J Neuroradiol 2004; 25: 1147-53.

8. Yamane F, Ishihara S, Kohyama S, et al. Local thrombus formation at the coil-parent artery interface during endovascular coil embolization of cerebral aneurysms. J Neurol Surg Part Cent Eur Neurosurg 2012; 73: 358-68.

9. Rahme RJ, Zammar SG, El Ahmadieh TY, et al. The role of antiplatelet therapy in aneurysm coiling. Neurol Res 2014; 36 383-8.

10. Bond KM, Brinjikji W, Murad MH, et al. Diffusion-weighted imaging-detected ischemic lesions following endovascular treatment of cerebral aneurysms: a systematic review and meta-analysis. Am J Neuroradiol 2017; 38: 304-9.

11. Martínez-Pérez R, Lownie SP, Pelz D. Intra-arterial use of abciximab in thromboembolic complications associated with cerebral aneurysm coiling: the london ontario experience. World Neurosurg 2017; 100: 342-50.

12. Edwards NJ, Jones WH, Sanzgiri A, et al. Antiplatelet therapy for the prevention of peri-coiling thromboembolism in highrisk patients with ruptured intracranial aneurysms. J Neurosurg 2017; 127: 1326-32.

13. Park HK, Horowitz M, Jungreis C, et al. Periprocedural morbidity and mortality associated with endovascular treatment of intracranial aneurysms. AJNR Am J Neuroradiol 2005; 26: 506-14.
14. Pierot L, Cognard C, Anxionnat R, et al. Ruptured intracranial aneurysms: factors affecting the rate and outcome of endovascular treatment complications in a series of 782 patients (CLARITY Study). Radiology 2010; 256: 916-23.

15. Lee SH, Jang MU, Kang J, et al. Impact of reducing the procedure time on thromboembolism after coil embolization of cerebral aneurysms. Front Neurol 2018; 9: 1125.

16. Kim KS, Fraser JF, Grupke S, Cook AM. Management of antiplatelet therapy in patients undergoing neuroendovascular procedures. J Neurosurg 2018; 129: 890-905.

17. Kocur D, Przybyłko N, Bażowski P, Baron J. Rupture during coiling of intracranial aneurysms: predictors and clinical outcome. Clin Neurol Neurosurg 2018; 165: 81-7.

18. Choi HH, Lee JJ, Cho YD, et al. Antiplatelet premedication for stent-assisted coil embolization of intracranial aneurysms: low-dose prasugrel vs clopidogrel. Neurosurgery 2018; 83: 981-8.

19. Adeeb N, Griessenauer CJ, Moore JM, et al. Ischemic stroke after treatment of intraprocedural thrombosis during stent-assisted coiling and flow diversion. Stroke 2017; 48: 1098-100.

20. Higashida RT, Furlan AJ. Trial design and reporting standards for intra-arterial cerebral thrombolysis for acute ischemic stroke. Stroke 2003; 34: e109-37.

21. Seo DH, Yoon SM, Park HR, et al. Thromboembolic event detected by diffusion weighted magnetic resonance imaging after coil embolization of cerebral aneurysms. I Cerebrovasc Endovasc Neurosurg 2014; 16: 175-83.

22. Choi HH, Cho YD, Yoo DH, et al. Stent-assisted coil embolization of anterior communicating artery aneurysms: safety, effectiveness, and risk factors for procedural complications or recanalization. J Neurointerv Surg 2019; 11: 49-56.

23. Altay T, Kang HI, Woo HH, et al. Thromboembolic events associated with endovascular treatment of cerebral aneurysms. J Neurointerv Surg 2011; 3: 147-50.

24. Bruening R, Mueller-Schunk S, Morhard D, et al. Intraprocedural thrombus formation during coil placement in ruptured intracranial aneurysms: treatment with systemic application of the glycoprotein IIb/IIla antagonist tirofiban. AJNR Am J Neuroradiol 2006; 27: 1326-31.

25. Ramakrishnan P, Yoo AJ, Rabinov JD, et al. Intra-arterial eptifibatide in the management of thromboembolism during endovascular treatment of intracranial aneurysms: case series and a review of the literature. Interv Neurol 2013; 2: 19-29.

26. Davis K, Morrison C. Ticagrelor for neuroendovascular procedures: a case series. J Pharm Pract 2018; 31: 115-9.

27. Hassan AE, Zacharatos $H$, Vazquez $G$, et al. Low risk of intracranial and systemic hemorrhages in patients on dual antiplatelet treatment beyond 1 month following neuroendovascular angioplasty and/or stent placement. J Neuroimaging 2012; 22: 67-73.

28. Cho YD, Lee JY, Seo JH, et al. Intra-arterial tirofiban infusion for thromboembolic complication during coil embolization of ruptured intracranial aneurysms. Eur J Radiol 2012; 81: 2833-8.

29. Hwang G, Huh W, Lee JS, et al. Standard vs modified antiplatelet preparation for preventing thromboembolic events in patients with high on-treatment platelet reactivity undergoing coil embolization for an unruptured intracranial 
aneurysm: a randomized clinical trial. JAMA Neurol 2015; 72: 764-72.

30. Sim SY, Shin YS. Silent microembolism on diffusion-weighted MRI after coil embolization of cerebral aneurysms. Neurointervention 2012; 7: 77-84

31. Kim B, Kim K, Jeon P, et al. Thromboembolic complications in patients with clopidogrel resistance after coil embolization for unruptured intracranial aneurysms. Am J Neuroradiol 2014; 35: 1786-92.

32. Sedat J, Chau Y, Gaudard J, et al. Administration of eptifibatide during endovascular treatment of ruptured cerebral aneurysms reduces the rate of thromboembolic events. Neuroradiology 2015; 57: 197-203.

33. Gralla J, Rennie ATM, Corkill RA, et al. Abciximab for thrombolysis during intracranial aneurysm coiling. Neuroradiology 2008; 50: 1041-7.

34. Ryu CW, Park S, Shin HS, Koh JS. Complications in stent-assisted endovascular therapy of ruptured intracranial aneurysms and relevance to antiplatelet administration: a systematic review. Am J Neuroradiol 2015; 36: 1682-8.

35. Brzegowy P, Kucybała I, Krupa K, et al. Angiographic and clinical results of anterior communicating artery aneurysm endovascular treatment. Videosurgery Miniinvasive Tech 2019; 14: 451-60.

36. Linfante I, Etezadi V, Andreone V, et al. Intra-arterial abciximab for the treatment of thrombus formation during coil embolization of intracranial aneurysms. J Neurointerv Surg 2010; 2: 135-8.

37. Kang HS, Kwon BJ, Roh HG, et al. Intra-arterial tirofiban infusion for thromboembolism during endovascular treatment of intracranial aneurysms. Neurosurgery 2008; 63: 230-8.

38. Kim HK, Hwang SK, Kim SH. Types of thromboembolic complications in coil embolization for intracerebral aneurysms and management. J Korean Neurosurg Soc 2009; 46: 226-31.

39. Demartini Junior Z, Gatto LAM, Koppe GL, et al. Rescue therapy with stent retrievers for thromboembolism during endovascular treatment of intracranial aneurysms. Arq Neuropsiquiatr 2018; 76: 332-8.

40. Koebbe CJ, Horowitz MB, Levy El, et al. Intraarterial thrombolysis for thromboemboli associated with endovascular aneurysm coiling: report of five cases. Interv Neuroradiol 2002; 8: 151-8.

41. Rha JH, Saver JL. The impact of recanalization on ischemic stroke outcome: a meta-analysis. Stroke 2007; 38: 967-73.

Received: 7.07.2019, accepted: 4.09.2019. 